\title{
Análisis de la pesquería del jurel Trachurus murphyi en el Perú
}

\author{
Analysis of the Jack mackerel Trachurus murphyi fishery in Peru
}

Miguel Ñiquen, Marilú Bouchon, Danny Ulloa y Ana Medina

Instituto del Mar del Perú, esquina Gamarra y General Valle S/N Chucuito, Callao, Perú.

Email Miguel Ñiquen: mniquen@imarpe.gob.pe

Email Marilú Bouchon: mbouchon@imarpe.gob.pe
Citación:

Ñiquen M., M. Bouchon, D. Ulloa y A. Medina. 2013. Análisis de la pesquería del jurel Trachurus murphyi en el Perú. En: Csirke J., R. Guevara-Carrasco \& M. Espino (Eds.). Ecología, pesquería y conservación del jurel pino (Eds.). Ecología, pesquería y conservación del jurel
(Trachurus murphyi) en el Perú. Rev. peru. biol. número especial 20(1): 097 - 106 (Septiembre 2013)

\section{Resumen}

La presencia del jurel Trachurus murphyi ha sido registrado en los desembarques de la pesca artesanal en Perú desde 1907. A partir del año 2000, las capturas de T. murphyi se ubicaron en el tercer lugar después de la anchoveta Engraulis ringens y el calamar gigante Dosidicus gigas. Las capturas de T. murphyi son realizadas por la flota artesanal, industrial cerquera y barcos arrastreros factoría. En el año 2011, la flota industrial cerquera con permiso de pesca para T. murphyi en la costa peruana estaba constituida por 62 embarcaciones industriales de acero $\left(30177 \mathrm{~m}^{3}\right.$ ) y 42 industriales de madera $\left(3082 \mathrm{~m}^{3}\right)$. Las capturas de T. murphyi tuvieron un notable incremento a partir de 1972, alcanzando sus máximos valores en 1977, 1996-97 y 2001, a los que siguieron años de muy baja disponibilidad que llevó al mínimo registro en las capturas en 2010 . Sin embargo en el 2011 se revirtió esta baja disponibilidad y las capturas alcanzaron casi las 260000 toneladas. En el periodo 1972 - 2012 se observó una relación inversa entre los estimados de biomasa acústica del jurel T. murphyi y los desembarques anuales de anchoveta $E$. ringens. Las capturas mensuales de T. murphyi por la flota industrial y artesanal antes del 2002 fueron altas en la zona norte de Perú (Mancora-Chimbote), mientras que después del 2002 las capturas fueron altas en la zona centro-sur (Huacho-Ilo).

Palabras clave: Jurel, recursos pelágicos, pesquería peruana, esfuerzo de pesca.

\section{Abstract}

There are records of the presence of Jack mackerel Trachurus murphyi in the artisanal fishery landings in Peru since 1907. Since 2000 Jack mackerel T. murphyi catches are ranked third after anchovy Engraulis ringens and jumbo squid Dosidicus gigas. Artisanal, industrial purse seine and offshore (factory trawlers) fleets take part in the Peruvian catches of $T$. murphyi. In 2011 the industrial purse-seine fleet with license to fish for T. murphyi along the Peruvian coast comprised of 62 industrial steel vessels $\left(30177 \mathrm{~m}^{3}\right)$ and 42 industrial wood vessels $\left(3082 \mathrm{~m}^{3}\right)$ Catches of T. murphyi had a significant increase after 1972, with maximum catches in 1977, 1996-97 and 2001, after which several years of low availability followed, with the lowest catches in 2010. However, this low availability trend was reversed in 2011, and catches reached almost 260 thousand tons that year. A general inverse relationship has been observed between the T. murphyi estimated acoustic biomass and the annual landings of anchovy $E$. ringens during the the period $1972-2012$. The monthly catches of T. murphyi by the industrial and artisanal fleets before 2002 were higher in the northern part of Peru (Mancora-Chimbote) while after 2002 the catches were higher in the south-central zone (Huacho-llo).

Keywords: Jack mackerel, pelagic, Peruvian fishery, fishing effort.

\section{Introducción}

El jurel, Trachurus murphyi Nichols 1920 es una especie transzonal de amplia distribución en el mar peruano. Es un recurso importante de la pesquería pelágica del Perú, que se caracteriza por ubicarse en diferentes condiciones oceanográficas y realiza desplazamientos a áreas favorables ante alteraciones climáticas (Tsukayama 1983, Zuta 1983, Bertrand et al. 2004).

Desde sus inicios, la extracción de T. murphyi ha sido realizada por diferentes tipos de flota, tanto en tamańo como en estrategias de pesca. Coker en el ańo 1907 reporta capturas de T. murphyi en el mar peruano. Sin embargo, los registros de desembarques empezaron en 1939 con cantidades que no superaban las 10 toneladas anuales. Esta
Publicado online: $\quad 11 / 10 / 2013$ Publicado impreso: $15 / 10 / 2013$ 
situación se mantuvo hasta 1962, año en el que se superaron las 1000 toneladas. Desde los años sesenta, las estadísticas ubican a T. murphyi entre las tres primeras especies de peces pelágicos en la pesquería e, inclusive a partir del año 2000 se ubicó en segundo lugar después de la anchoveta.

Antes de 2002, cerca de 500 embarcaciones de un total aproximado de 1200 que conformaban la flota de cerco industrial dirigieron su pesca al jurel T. murphyi, principalmente en periodos de veda de la anchoveta Engraulis ringens. En su mayoría, estas embarcaciones contaban con aparejos de pesca y sistemas de conservación que les permitían alternar entre una y otra espacie pelágica.

El año 2002 se promulgó el DS Nº 001-2002-PRODUCE, que estableció el uso exclusivo para consumo humano directo del jurel T. murphyi junto con la sardina Sardinops sagax y la caballa Scomber japonicus. Ese decreto limitó las capturas y permitió el desarrollo de una flota industrial con sistema de refrigeración RSW (Refrigerated Sea Water), con características especiales para consumo humano directo. Posteriormente, el 12 de abril del 2007 se estableció el Reglamento de Ordenamiento Pesquero de Jurel y Caballa, aprobado por DS Nº. 011-2007-PRODUCE, que norma y promueve la explotación racional de los recursos jurel $T$. murphyi y caballa $S$. japonicus en un contexto de conservación y sostenibilidad de estos recursos.

El presente trabajo describe el comportamiento del esfuerzo de pesca y las capturas de T. murphyi efectuadas en Perú, desde 1939 a 2012, y de esta manera proporciona la información básica para la evaluación del stock existente en el mar peruano.

\section{Material y métodos}

La zona de estudio comprende todo el mar peruano, desde el extremo norte hasta el extremo sur del dominio marítimo y desde la línea de costa hasta las 200 millas, considerándose tres regiones: Norte $\left(3^{\circ} 24^{\prime}-09^{\circ} 59^{\prime} S\right)$, Centro $\left(10^{\circ}-15^{\circ} 59^{\prime} S\right)$ y Sur $\left(16^{\circ} \mathrm{S}\right.$ - Extremo Sur).

El análisis está basado en las capturas mensuales (toneladas) de toda la serie histórica de la pesquería de $T$. murphyi, tanto de la flota industrial como de la artesanal. Para el periodo comprendido entre 1939 - 1962 se consideró la serie obtenida por Tilic (1963), y a partir de 1963 hasta el 2012 los datos fueron obtenidos de los Anuarios Pesqueros del Ministerio de Producción, complementados con informaciones del IMARPE basadas en datos del Seguimiento de la Pesquería Pelágica para la pesca industrial y la Unidad de Estadística para la pesca artesanal. En el caso del esfuerzo pesquero se utilizó el número de embarcaciones, reconstruido en base a informaciones obtenidas por Bouchon et al. (2000).

Los datos de condiciones ambientales en el mar peruano provienen de cruceros de evaluación hidroacústica de recursos pelágicos ejecutados durante el periodo 1983 - 2012 con los BIC Olaya y SNP-2, cubriendo el área comprendida entre la costa y $100 \mathrm{mn}$ del dominio marítimo peruano. Los datos mensuales de temperatura superficial del mar (TSM) provienen de los registros efectuados en el puerto de Chicama por el IMARPE.

\section{Resultados y discusión}

Flota: estructura y distribución (cerco y arrastre).- En el periodo comprendido entre los ańos 1970 a 2012 de las diferentes flotas (artesanal, industrial de cerco y de arrastre factoría) que actuaron sobre los recursos jurel T. murphyi y caballa S. japonicus,

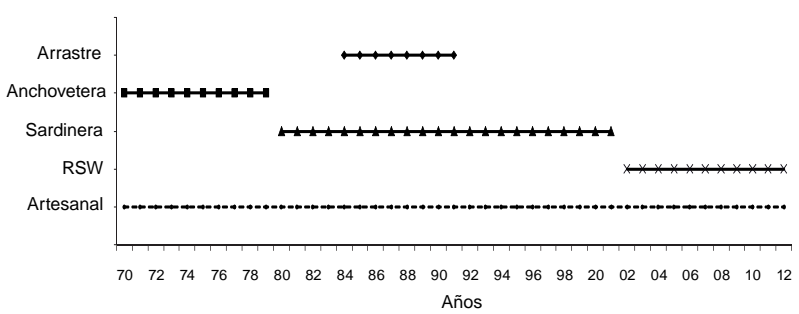

Figura 1. Periodos de operación de las diversas flotas pesqueras dirigidas a la captura de jurel T. murphyi en el mar peruano durante los años 1970-2012 (RSW = con sistema de refrigeración por agua de mar refrigerada, del inglés Refrigerated Sea Water)

Figure. 1. Periods of operation of the various fishing fleets targeting on Jack mackerel T. murphyi in Peruvian waters during 1970-2012 (RSW = Refrigerated Sea Water)

destacó la actividad permanente de la flota artesanal y la flota cerquera, que presento variaciones en su estructura pasando de ser una flota anchovetera en sus inicios hacia una flota sardinera que impulsó la captura de T. murphyi para el consumo humano directo, situación que actualmente se realiza mediante las actividades de la flota con sistema de refrigeración RSW (Fig. 1).

La flota artesanal emplea embarcaciones de pequeño calado, que cuentan hasta con $30 \mathrm{~m}^{3}$ de capacidad de bodega y operan en la zona costera cerca a los puertos y caletas de origen. La flota industrial está conformada por todas aquellas embarcaciones mayores o iguales a $32.6 \mathrm{~m}^{3}$ de capacidad de bodega, comúnmente conocidas como "bolicheras", que usan redes de cerco, algunas de ellas con sistema de refrigeración a bordo. Estas embarcaciones capturan incidentalmente el jurel T. murphyi, pues normalmente se dedican a la pesquería de sardina $S$. sagax o anchoveta $E$. ringens. Esta flota estuvo constituida por 500 embarcaciones, cuyas capacidades de bodega fluctuaron entre 32.6 y $800 \mathrm{~m}^{3}$, con un promedio de $199 \mathrm{~m}^{3}$ y una capacidad total acumulada de aproximadamente $99600 \mathrm{~m}^{3}$. Aquéllas que tienen permiso para pescar anchoveta $E$. ringens (flota anchovetera) emplean redes con abertura de malla de $13 \mathrm{~mm}$ ( $1 / 2$ pulgada), mientras las que tienen permiso múltiple tanto para anchoveta $E$. ringens como para sardina $S$. sagax, jurel T. murphyi y caballa S. japonicus (flota sardinera), cuando se dirigen a pescar $T$. murphyi emplean redes con abertura de malla de $38 \mathrm{~mm}$ ( 1 y $1 / 2$ pulgada).

Por otra parte, durante los ańos 1984 - 1991, mediante convenios especiales con países como la ex-URSS y Cuba, se permitió la operación de buques arrastreros factoría (BAF). Esta flota arrastrera de media agua operó generalmente fuera de las 20 millas de la costa en la región norte, entre Paita y Chimbote, contando con 6 a 14 barcos pertenecientes a la flota cubana y entre 22 a 26 barcos pertenecientes a la flota de la ex-URSS (Tabla 1). Al inicio de esta pesquería esta flota presentó sus mejores rendimientos con capturas de $561 \mathrm{t} / \mathrm{barco} / \mathrm{mes}$.

En la década de los setenta, la flota cerquera pelágica presentó un comportamiento irregular debido principalmente a la inestabilidad y reducción de la población de anchoveta $E$. ringens, que trajo como consecuencia la disminución considerable del número de embarcaciones. Esta situación empezó a revertirse a partir de 1985 y en la década de los noventa la flota cerquera pelágica aumentó en número y capacidad de bodega. Además se observó un importante desarrollo tecnológico de la flota, registrándose variaciones en su composición, renovación de los equipos de pesca, modificaciones en las redes, inclusión de 
Tabla 1. Periodos de operación de barcos arrastreros factoría y sus capturas de jurel T. Murphyi en Perú, $1984-1991$

Table 1. Operative periods of factory trawlers and their catches of Jack mackerel T. murphyi in Peru, $1984-1991$

\begin{tabular}{lccccc}
\hline Periodo & $\begin{array}{c}\text { Nacionalidad de la } \\
\text { flota }\end{array}$ & $\begin{array}{c}\text { Número de meses } \\
\text { operativos }\end{array}$ & $\begin{array}{c}\text { Número de } \\
\text { barcos }\end{array}$ & $\begin{array}{c}\text { Captura jurel } \\
\text { (toneladas) }\end{array}$ & $\begin{array}{c}\text { Toneladas de jurel/ } \\
\text { barco/mes }\end{array}$ \\
\hline Mayo 1984 - agosto 1985 & ex-URSS & 16 & 22 & 197621 & 561 \\
Enero 1986 - marzo 1987 & Cuba & 15 & 06 & 37774 & 420 \\
Enero - diciembre 1988 & Cuba & 12 & 14 & 45656 & 272 \\
Enero 1989 - mayo 1991 & ex-URSS & 29 & 26 & 227191 & 301 \\
\hline
\end{tabular}

algún sistema de refrigeración y mayor autonomía y capacidad de desplazamiento hacia las áreas y núcleos de concentración, lo cual significó un incremento en el poder de pesca de la flota (Bouchon et al. 2000, Niquen et al. 2000).

Características de la flota cerquera.- La flota cerquera que opera en el mar peruano está conformada por embarcaciones con un amplio rango de capacidad de bodega que va desde 100 hasta las $868 \mathrm{~m}^{3}$, con un promedio de $500 \mathrm{~m}^{3}$. La cobertura de acción de la flota peruana dirigida a la pesca de T. murphyi se caracteriza por ser costera, pues en promedio las actividades de extracción se realizan en la franja de las 100 primeras millas náuticas. En la Tabla 2 se presentan algunas características de la flota cerquera dedicada a la pesca de T. murphyi.

En el año 2006 la estructura de la flota con permiso de pesca de T. murphyi para consumo humano directo en la costa peruana contó con la participación de embarcaciones de la flota industrial

Tabla 2. Características de la flota cerquera dedicada a la pesca de jurel T. murphyi.

Table 2. Features of the purse seine fleet devoted to fish for Jack mackerel T. murphyi.

\begin{tabular}{rc}
\hline \multicolumn{1}{c}{ Características } & Barco Cerquero (flota Perú) \\
\hline Artes de pesca (método) & Activa (Niveles superficiales) \\
Tipo de pesca & Embolsamiento \\
Materiales redes & Tenaz, alta densidad \\
Cardúmenes & Plumas o concentraciones mayores, \\
superficiales \\
Volúmenes & más de 500 t por lance \\
Cobertura & Costera \\
Días de operación efectiva & $4-5$ \\
Número de lances por día & $01-04$ \\
Procesos de la captura & Fresco en agua refrigerada \\
Sistema de frío & Agua de mar refrigerada (RSW). \\
& Agua de mar enfriada (CSW) \\
\hline
\end{tabular}

de acero y de la flota industrial de madera. El número total de embarcaciones fue de 542, de las cuales 84 fueron industriales de acero y 458 industriales de madera. La capacidad de bodega acumulada de toda la flota con permiso para jurel fue de 50587 $\mathrm{m}^{3}$, de las cuales $36488 \mathrm{~m}^{3}$ corresponden a la flota industrial de acero y $28892 \mathrm{~m}^{3}$ a la flota industrial de madera.

A partir de 2011 se redujo la flota con permiso de pesca para T. murphyi en la costa peruana a 104 embarcaciones, de las cuales 62 son industriales de acero y 42 industriales de madera. La capacidad de bodega acumulada de toda la flota con permiso para T. murphyi es de $33359 \mathrm{~m}^{3}$, de las cuales 30177 $\mathrm{m}^{3}$ corresponden a la flota industrial de acero y $3082 \mathrm{~m}^{3}$ a la flota industrial de madera (Tabla 3).

Tabla 3. Número y capacidad de bodega $\left(\mathrm{m}^{3}\right)$ de la flota operativa dirigida al jurel T. murphyi usando red de cerco durante el 2011.

Table 3. Number of vessels and hold capacity $\left(\mathrm{m}^{3}\right)$ of the operational fleet targeting on Jack mackerel T. murphyi with purse seine during 2011.

\begin{tabular}{cccc}
\hline \multicolumn{4}{l}{ Flota industrial de madera (usando sistema de cajas/hielo) } \\
\hline $\begin{array}{c}\text { Rango de } \\
\text { capacidad } \\
\text { de bodega } \\
\left(\mathrm{m}^{3}\right)\end{array}$ & $\begin{array}{c}\text { Número } \\
\text { barcos }\end{array}$ & $\begin{array}{c}\text { Total de la } \\
\text { capacidad de } \\
\text { bodega }\left(\mathrm{m}^{3}\right)\end{array}$ & $\begin{array}{c}\text { Promedio de la } \\
\text { capacidad de } \\
\text { bodega }\left(\mathrm{m}^{3}\right)\end{array}$ \\
\hline $31-60$ & 14 & 556 & 39.7 \\
$61-90$ & 13 & 991 & 76.2 \\
$91-110$ & 15 & 1535 & 102.3 \\
Total & 42 & 3082 & 73.4 \\
\hline Flota industrial de acero (usando sistema RSW) \\
\hline $111-300$ & 2 & 348 & 174.0 \\
$301-400$ & 6 & 2167 & 361.2 \\
$401-500$ & 29 & 12913 & 445.3 \\
$501-600$ & 19 & 10537 & 554.6 \\
$601-700$ & 4 & 2477 & 619.3 \\
$>700$ & 2 & 1735 & 867.5 \\
Total & 62 & 30177 & 486.7 \\
\hline
\end{tabular}

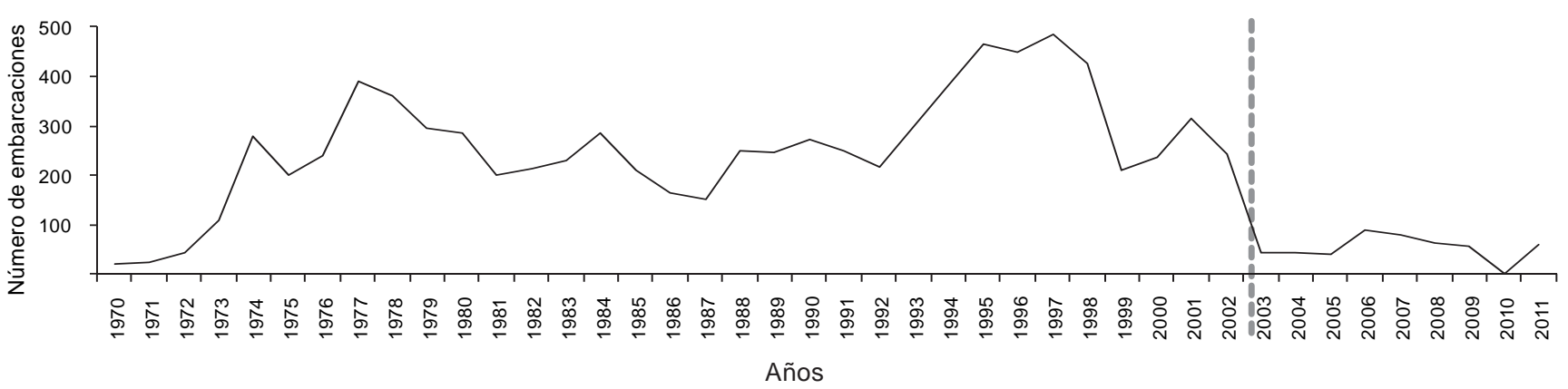

Figura 2. Número de embarcaciones operativas de la flota de cerco en la pesquería de jurel T. murphyi frente a Perú durante 1970 - 2011 (la línea punteada indica fecha en que el jurel comienza a ser usado exclusivamente para consumo humano directo)

Figure 2. Number of operational vessels of the purse seine fleet in the Peruvian Jack mackerel T. murphyi fishery during $1970-2011$ (the dotted line indicates the date that the Jack mackerel begin to be used exclusively for direct human consumption) 
Tabla 4. Comentarios sobre presencia de jurel en Perú (de Coker 1907, 1910).

Table 4. Comments on the presence of Jack mackerel in Peru (from Coker 1907, 1910).

\begin{tabular}{|c|c|}
\hline Localidad & Comentarios sobre presencia de jurel \\
\hline Paita & Siempre abundante \\
\hline Bahía de Sechura & Presencia de jureles \\
\hline \multirow[t]{2}{*}{ Lobos de Tierra } & $\begin{array}{l}\text { Abundan por épocas. Los pescadores } \\
\text { cogieron ejemplares grandes que tenían } \\
\text { cerca de un metro de largo. }\end{array}$ \\
\hline & $\begin{array}{l}\text { Se observó un cardumen constituido } \\
\text { por un escaso número de peces, que } \\
\text { eran visiblemente jureles. }\end{array}$ \\
\hline Callao & $\begin{array}{l}\text { Se les considera regulares. Se presentan } \\
\text { todo el año y abundan en verano. Se les } \\
\text { saca con redes, chichorro y cordel. }\end{array}$ \\
\hline Chilca & $\begin{array}{l}\text { Abundantes, pescados con cordeles y } \\
\text { redes. }\end{array}$ \\
\hline Cerro Azul & $\begin{array}{l}\text { El jurel es uno de los principales } \\
\text { pescados de esta región. }\end{array}$ \\
\hline Pisco & $\begin{array}{l}\text { Anteriormente eran más abundantes } \\
\text { que hoy. Se les considera buenos. }\end{array}$ \\
\hline Mollendo & Es abundante. \\
\hline
\end{tabular}

El número de embarcaciones operativas de la flota industrial cerquera que participaron en la pesquería de T. murphyi durante el periodo 1970 - 2011 se presenta en la Figura 2. Este número alcanza un máximo cercano a las 500 embarcaciones en la década de los años noventa y se destaca que ha sido mucho menor y se ha mantenido casi constante desde el 2002 (Fig. 2).

Capturas de jurel T. murphyi: análisis de largo plazo y tendencia.- La extracción de T. murphyi se ha desarrollado en el Perú de manera intermitente, tanto como pesca objetivo o como pesca incidental de la pesquería de E. ringens.

En Coker (1907) se describió el estado de la pesquería en Perú durante 1907, explicando que "no hay en el mundo otras aguas que estén más copiosamente provistas de pequeños pescados que las del Perú, favorecidas por las enormes bandadas de anchovetas" (p. 95). Coker $(1907,1910)$ realizó un estudio sobre la pesca marina desde Paita $\left(05^{\circ} \mathrm{S}\right)$ hasta Mollendo $\left(17^{\circ} \mathrm{S}\right)$ y presentó un listado de los peces y mariscos existentes, donde destaca la importante presencia de jurel T. murphyi, según lo descrito en la Tabla 4.

En 1972 se observó un notable incremento con capturas superiores a las 10000 t, que llegaron a alcanzar en 1977 uno de
Tabla 5. Desembarques anuales de jurel Trachurus murphyi (toneladas) según flotas 1939-2012.

Table 5. Annual landings of Jack mackerel Trachurus murphyi (tonnes) according to fleets $1939-2012$

\begin{tabular}{|c|c|c|c|c|c|}
\hline Año & Cerco & Arrastre & Año & Cerco & Arrastre \\
\hline 1939 & 10 & & 1976 & 54154 & \\
\hline 1940 & 34 & & 1977 & 504992 & \\
\hline 1941 & 46 & & 1978 & 386793 & \\
\hline 1942 & 15 & & 1979 & 151591 & \\
\hline 1943 & 13 & & 1980 & 123380 & \\
\hline 1944 & 23 & & 1981 & 37875 & \\
\hline 1945 & 17 & & 1982 & 50013 & \\
\hline 1946 & 43 & & 1983 & 43433 & 33392 \\
\hline 1947 & 59 & & 1984 & 54607 & 129726 \\
\hline 1948 & 44 & & 1985 & 40717 & 46749 \\
\hline 1949 & 48 & & 1986 & 37006 & 31156 \\
\hline 1950 & 30 & & 1987 & 31588 & 14716 \\
\hline 1951 & 89 & & 1988 & 72420 & 45656 \\
\hline 1952 & 81 & & 1989 & 57516 & 83204 \\
\hline 1953 & 69 & & 1990 & 90544 & 100595 \\
\hline 1954 & 62 & & 1991 & 92212 & 44125 \\
\hline 1955 & 138 & & 1992 & 93065 & \\
\hline 1956 & 673 & & 1993 & 121309 & \\
\hline 1957 & 366 & & 1994 & 213220 & \\
\hline 1958 & 173 & & 1995 & 386748 & \\
\hline 1959 & 448 & & 1996 & 362197 & \\
\hline 1960 & 281 & & 1997 & 371485 & \\
\hline 1961 & 174 & & 1998 & 314123 & \\
\hline 1962 & 666 & & 1999 & 82541 & \\
\hline 1963 & 1954 & & 2000 & 296581 & \\
\hline 1964 & 1718 & & 2001 & 774603 & \\
\hline 1965 & 2561 & & 2002 & 154218 & \\
\hline 1966 & 4271 & & 2003 & 217735 & \\
\hline 1967 & 3071 & & 2004 & 187368 & \\
\hline 1968 & 2790 & & 2005 & 80663 & \\
\hline 1969 & 4176 & & 2006 & 277567 & \\
\hline 1970 & 4711 & & 2007 & 254427 & \\
\hline 1971 & 9191 & & 2008 & 169537 & \\
\hline 1972 & 18782 & & 2009 & 74694 & \\
\hline 1973 & 42781 & & 2010 & 17559 & \\
\hline 1974 & 129211 & & 2011 & 257241 & \\
\hline 1975 & 37899 & & 2012 & 168779 & \\
\hline
\end{tabular}

sus mayores registros con $505000 \mathrm{t}$ (Fig. 3). Posteriormente, las capturas se mantuvieron entre 100 a 200 mil toneladas anuales, destacando los pulsos de 1984 y 1990, seguidos de un fuerte incremento en 1997 con 650000 t, hasta alcanzar su mayor captura en el 2001 con 723000 t (Fig. 3).

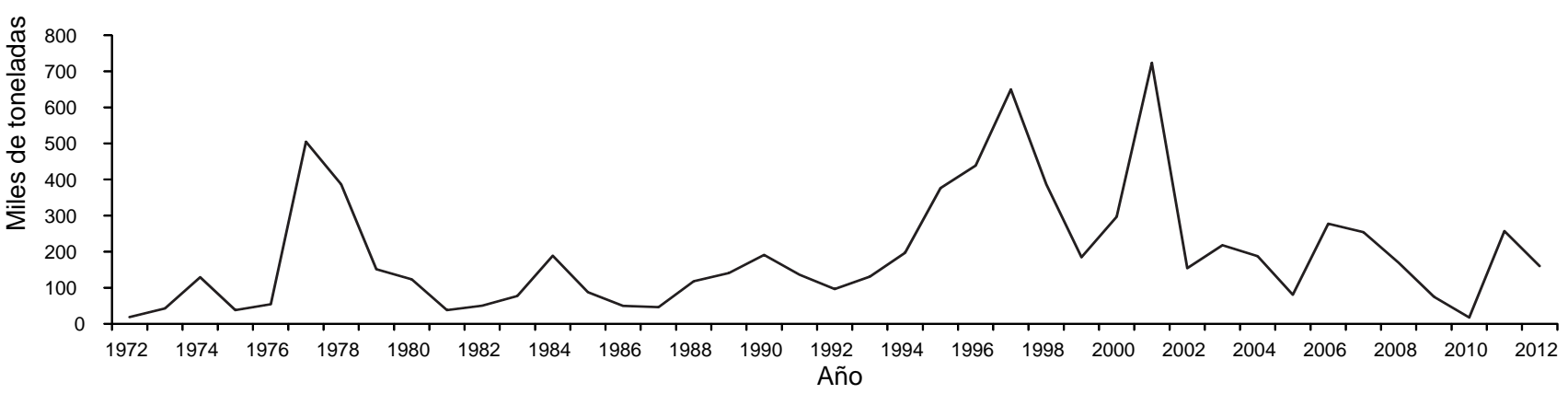

Figura 3. Desembarques anuales de jurel T. murphyi en Perú durante 1972 - 2012.

Figure 3. Annual landings of Jack mackerel T. murphyi in Peru during 1972 - 2012. 

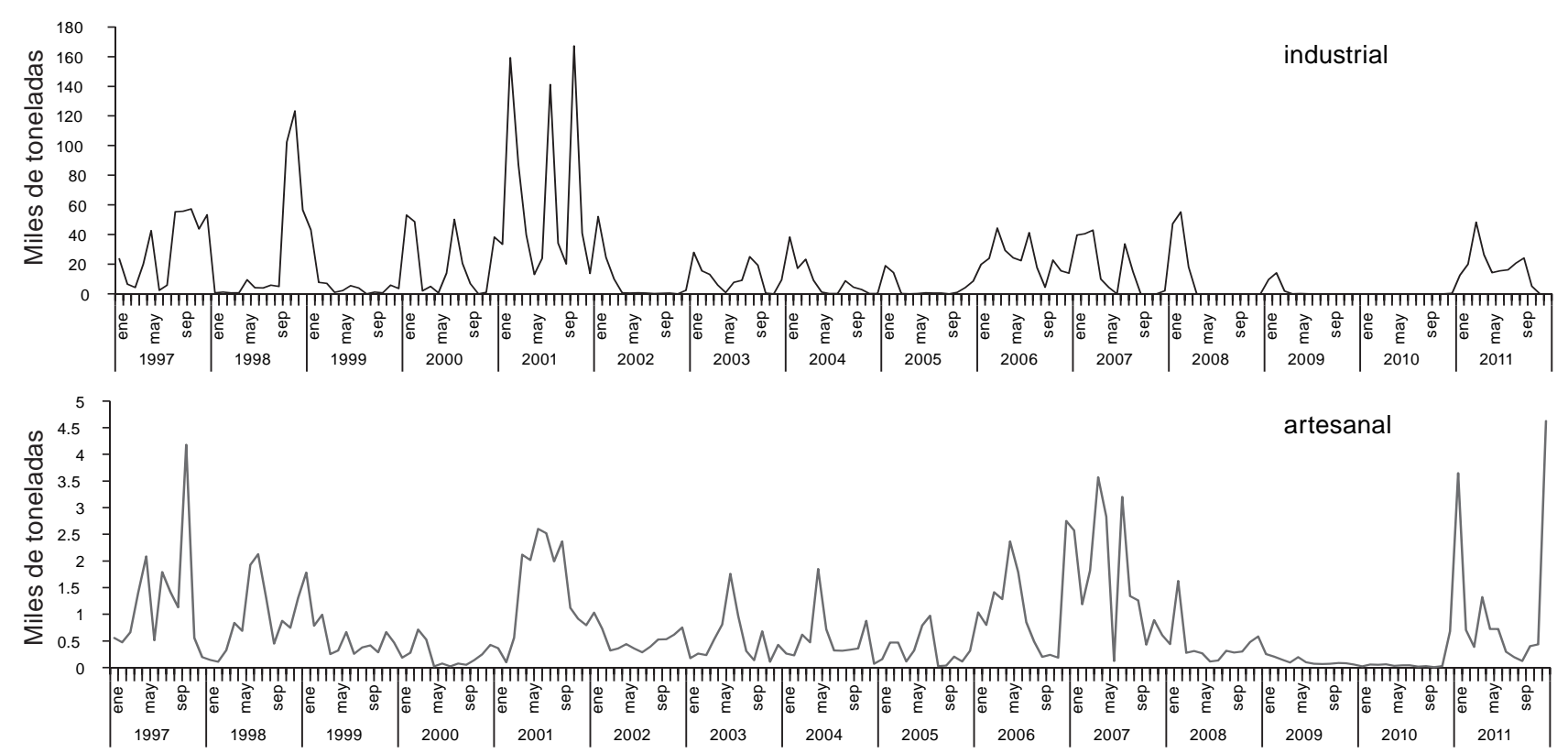

Figura 5. Captura mensual de jurel T. murphyi por: a) flota industrial y b) flota artesanal en el periodo $1997-2011$.

Figure 5. Monthly catch of Jack mackerel T. murphyi by: a) industrial fleet and b) artisanal fleet in the period 1997 - 2011

En el periodo 2002 - 2011 los reportes sobre desembarques anuales de T. murphyi en el litoral peruano presentaron una clara tendencia a disminuir desde el 2007 y tuvieron un registro mínimo en el 2010, debido a una baja disponibilidad de dicha especie (Fig. 3). Sin embargo, en el 2011 incremento su disponibilidad, lo cual representa un cambio en la tendencia decreciente de su captura, pues alcanzó una cifra cercana a las $260000 \mathrm{t}$, superior a lo obtenido en los años 2007 y 2008 (Tabla 5, Fig. 3).

En el periodo 1970 - 2002 se observó una marcada estacionalidad en las capturas de T. murphyi (Fig. 4), con mejores rendimientos en verano y primavera. Debemos destacar que en los últimos años $(2003-2011)$ las mayores capturas han ocurrido con más frecuencia en verano.

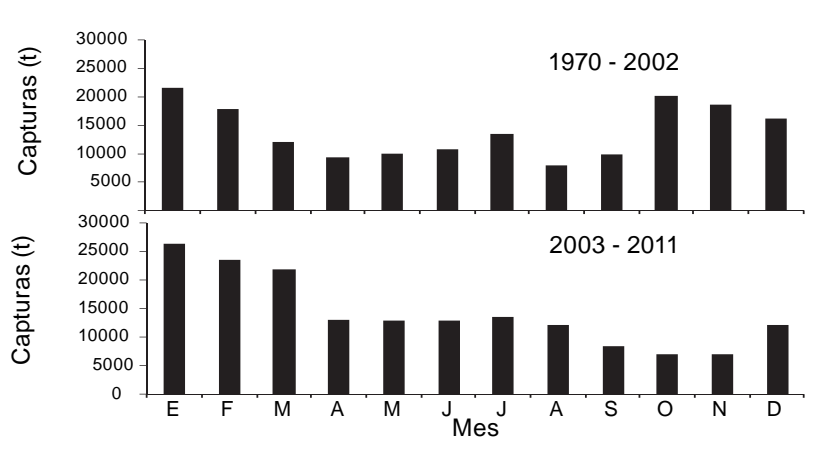

Figura 4. Estacionalidad de capturas de jurel T. murphyi en Perú, basada en promedios mensuales, periodos 1970-2002 y 2003-2011.

Figure 4. Catch seasonality of the Jack mackerel $T$. murphyi in Peru, based on monthly averages for two periods, 1970-2002 and 2003-2011.
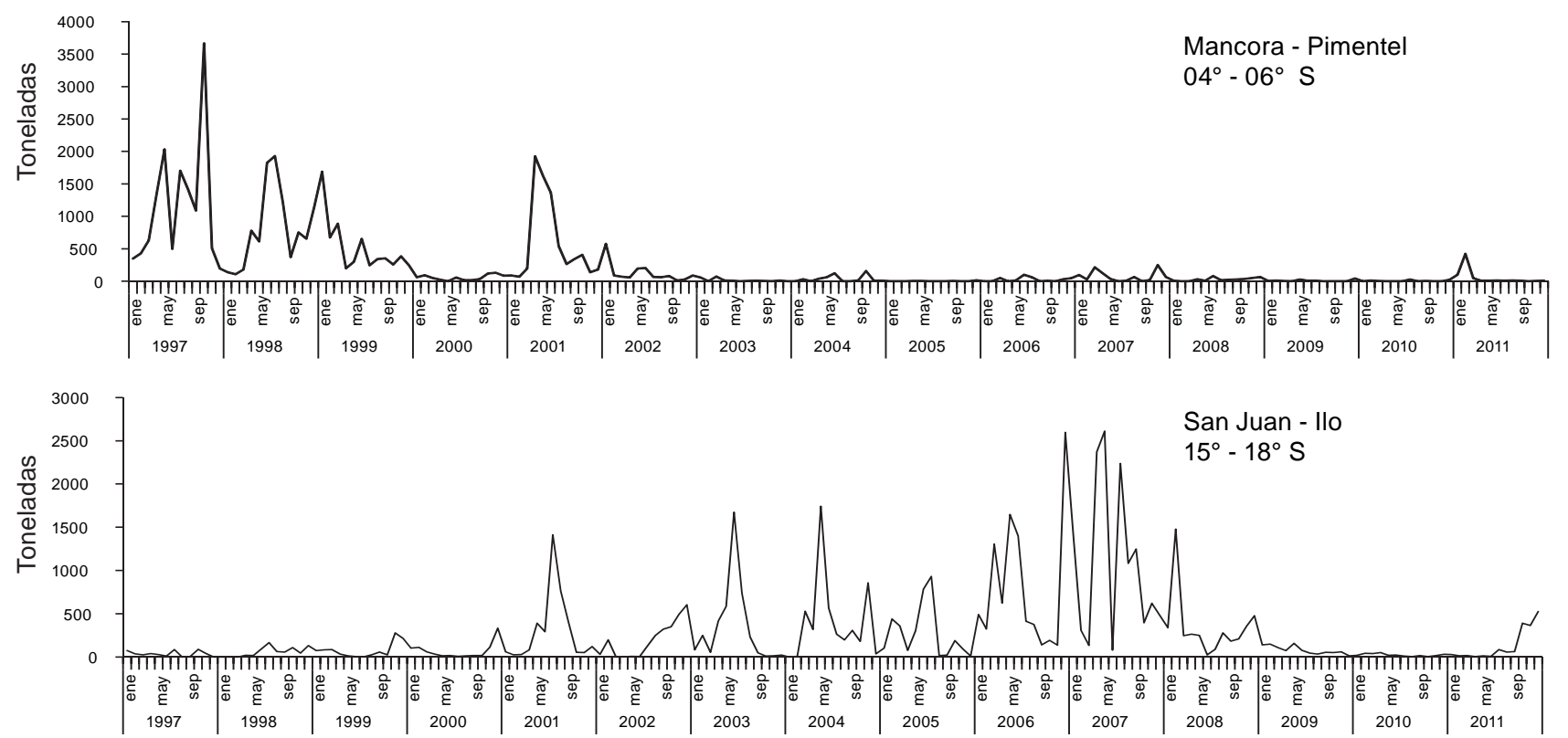

Figura 6. Capturas de jurel T. murphyi por la flota artesanal en el periodo 1997 - 2011, según zonas.

Figure 6. Jack mackerel T. murphyi catches by the artisanal fleet in the period $1997-2011$, by zones. 


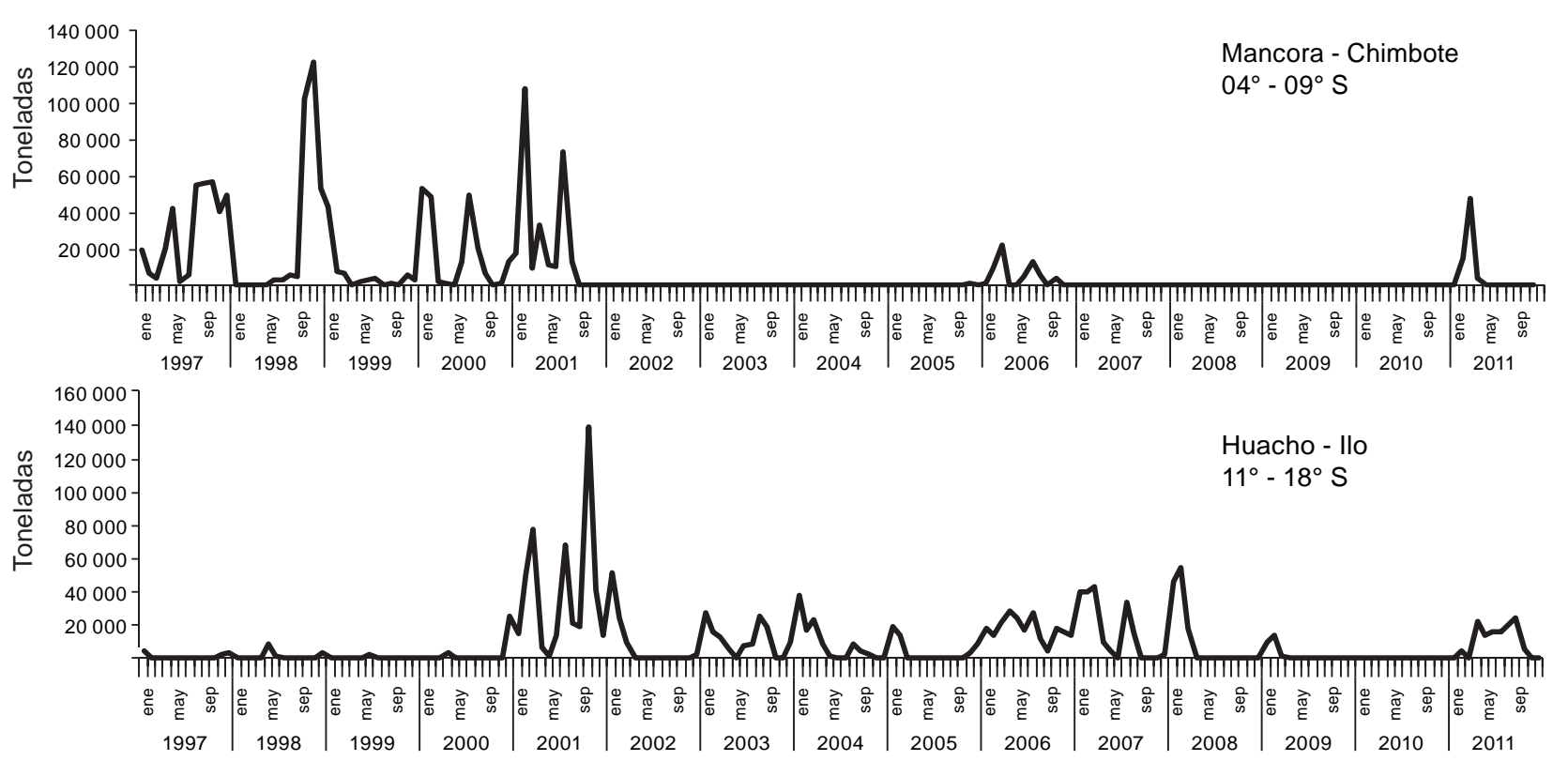

Figura 7. Capturas de jurel T. murphyi por la flota industrial en el periodo 1997 - 2011, según zonas.

Figure 7. Jack mackerel T. murphyi captures by the industrial fleet in the period $1997-2011$ by zones.

La información mensual sobre capturas de T. murphyi efectuadas por la flota industrial y la flota artesanal en el periodo 1997 - 2011 (Fig. 5a y 5b) mostró una tendencia similar, con periodos de mayor disponibilidad en el 2001 y entre los años 2006 a 2008. Los principales puntos de desembarque para la flota artesanal fueron Ilo, Pisco, Huacho, Matarani, Paita, Talara, Callao y Chimbote, mientras que en el caso de la flota industrial fueron Chimbote, Callao, Paita y Pisco.

Esta información también permitió observar variaciones en las principales zonas y niveles de pesca de $T$. murphyi de las flotas artesanal (Fig. 6) e industrial (Fig. 7). En ambos casos se observa que las capturas de T. murphyi en la parte norte (Máncora-Pimentel y Máncora-Chimbote) fueron mayores antes del 2002. Lo inverso sucedió en la parte centro sur (San Juan-Ilo y Huacho-Ilo), donde las capturas fueron mayores despues del 2001.

La información biológica provenientes de las capturas efectuadas por la flota cerquera dirigida al recurso jurel muestra una constante presencia del jurel T. murphyi y la caballa S. japonicus, lo que significaría que ambas comparten el mismo hábitat en rangos de tamaños similares. Durante el periodo 1970 - 2012, la información de los desembarques anuales efectuados por la flota de cerco industrial sobre composición por especies de jurel T. murphyi y caballa S. japonicus (Fig. 8) muestra que hubo predominancia de T. murphyi, en un promedio de $72 \%$ respecto al $28 \%$ de la caballa S. japonicus.

Cambios en la disponibilidad de jurel T. murphyi en función a variables ambientales.- Desde los primeros registros sobre la disponibilidad de T. murphyi en el Perú hasta el año 1996, tuvo una amplia distribución en todo el mar peruano, con estimaciones de biomasa acústica promedio anual de 4.8 millones de toneladas y promedio anual de desembarques de 142000 t. Sin embargo, la información posterior a 1996 muestra una distribución restringida a la parte centro-sur del mar peruano, con estimaciones de biomasa acústica promedio anual de $420000 \mathrm{t}$ y promedio anual de desembarques de $256000 \mathrm{t}$.

Una característica ambiental común observada en los ańos de mejor pesca de T. murphyi (1977, 1996-97 y 2001) fue la incidencia de anomalías negativas de la temperatura superficial del mar (Fig. 9). Esta incidencia de bajas temperaturas no fue

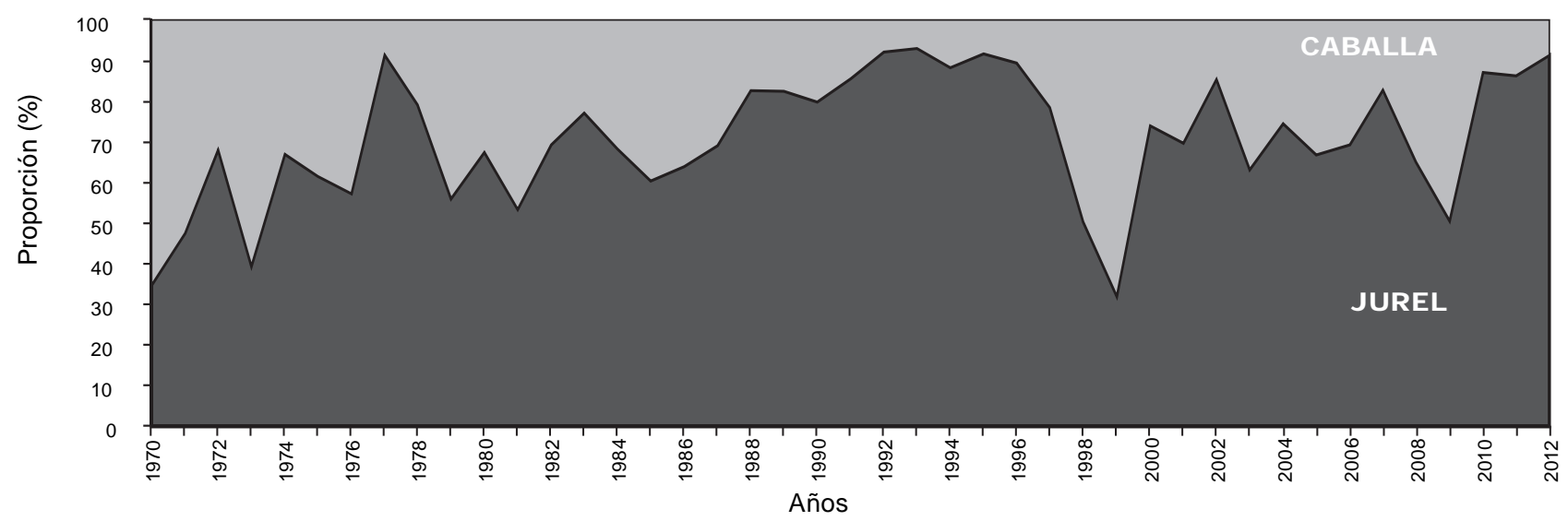

Figura 8. Composición porcentual de las capturas de jurel T. murphyi y caballa S. japonicus por años, periodo 1970 - 2012.

Figure 8. Percentage composition of the catches of Jack mackerel T. murphyi and mackerel S. japonicus by year, period $1970-2012$. 


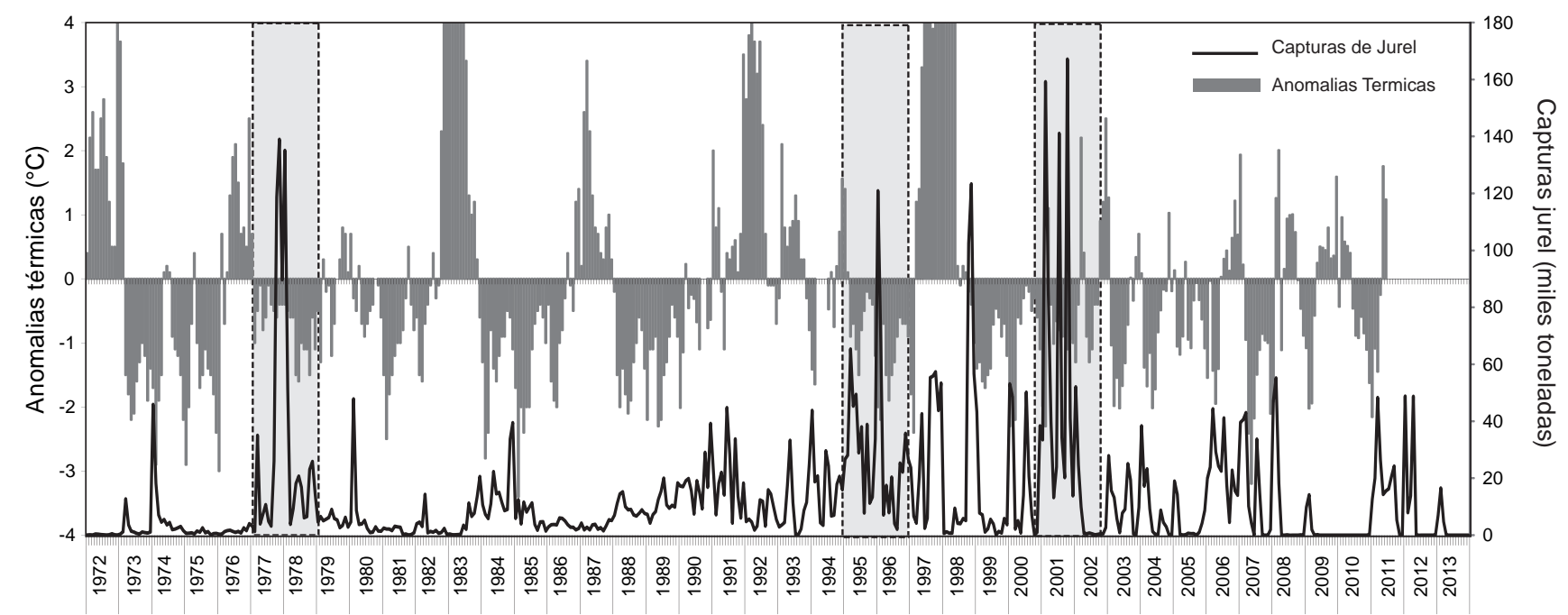

Años

Figura 9. Capturas mensuales de jurel T. murphyi en relación a las anomalías mensuales de la Temperatura Superficial del Mar (TSM) en Chicama durante 1972 - 2012. El área sombreada resalta la asociación entre la anomalía negativa de la TSM y la mayor disponibilidad de jurel. Figure 9. Monthly catches of Jack mackerel T. murphyi in relation to the monthly anomalies of the SuperfaceSea Temperature (SST) in Chicama during $1972-2012$. The shaded area highlights the association between negative anomaly of the SST and the greater availability of Jack mackerel.

de corto plazo, sino que se observo en varios ańos seguidos (1976 a 1978, de 1994 a mediados de 1997, y de 1999 al 2001). Estas condiciones favorables para la captura de $T$. murphyi podemos observarla en particular en el año 2001. En este año predominaron anomalías térmicas negativas al sur del Callao, asociadas a la presencia de las Aguas Templadas de la Subantártica y durante este año se observó la ocurrencia de intensos afloramientos y la intromisión de Aguas Subtropicales Superficiales, características ambientales que favorecieron la disponibilidad de T. murphyi. La persistencia del enfriamiento en estos periodos favoreció la presencia de masas de agua homogéneas que permitieron un mayor desplazamiento de los cardúmenes de T. murphyi, tanto de sur a norte como de oeste a este, haciéndolos más disponibles a la acción de la flota cerquera pelágica (Gutierrez et al. 2004).

Simultáneamente a la mayor disponibilidad de T. murphyi en los años 1977, 1996-97 y 2001, se observó una menor disponibilidad de E. ringens (Fig. 10). Esta relación inversa se explica por la tendencia de la anchoveta $E$. ringens a concentrarse hacia la región norte, mientras que el jurel T. murphyi se concentra en las regiones centro-sur en estos periodos, haciéndose más disponible a la acción de la flota cerquera pelágica. Esta relación inversa entre las capturas de T. murphyi y E. ringens también fue registrada en los estimados de biomasa acústica en el mar peruano durante el periodo 1983 -2012, observándose además que después de 1995 dominó claramente $E$. ringens y disminuyó las biomasas de jurel T. murphyi (Fig. 11).

El jurel T. murphyi y la caballa $S$. japonicus son recursos que habitan distintos espacios de altamar y zonas costeras, en particularmente en los frentes oceánicos, que son zonas productivas donde se encuentran dos tipos de masas de agua (Grechina 1998). En particular, T. murphyi es una especie cuya distribución frente al Perú, en la columna de agua, está asociada a la profundidad de la capa de mínimo oxígeno y a las Aguas Subtropicales Superficiales (ASS) (Dioses 2013). Los indicadores ambientales de largo plazo seńalan que la profundidad de la capa de mínimo oxígeno (Fig. 12) ha sufrido cambios decadales frente a la costa peruana (Bertrand

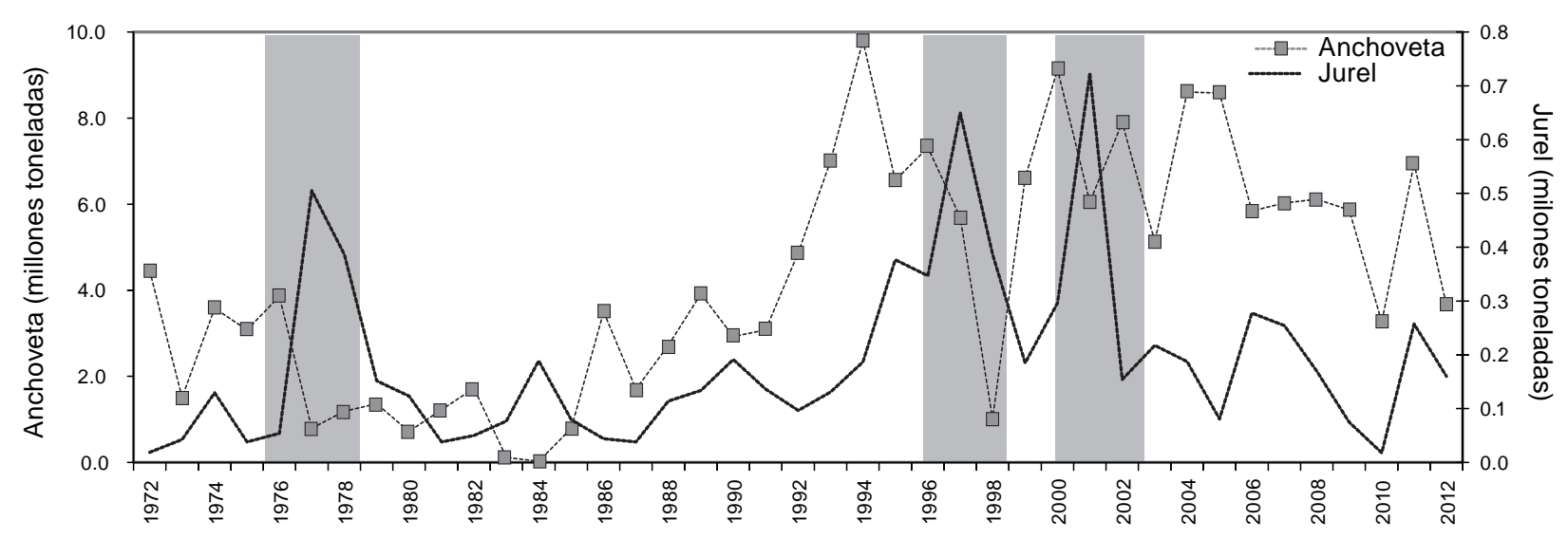

Figura 10. Desembarques anuales de jurel T. murphyi en relación con los desembarques de anchoveta durante 1972 - 2012.

Figure 10. Annual ladings of Jack mackerel T. murphyi in relation with the landings of anchovy during $1972-2012$. 


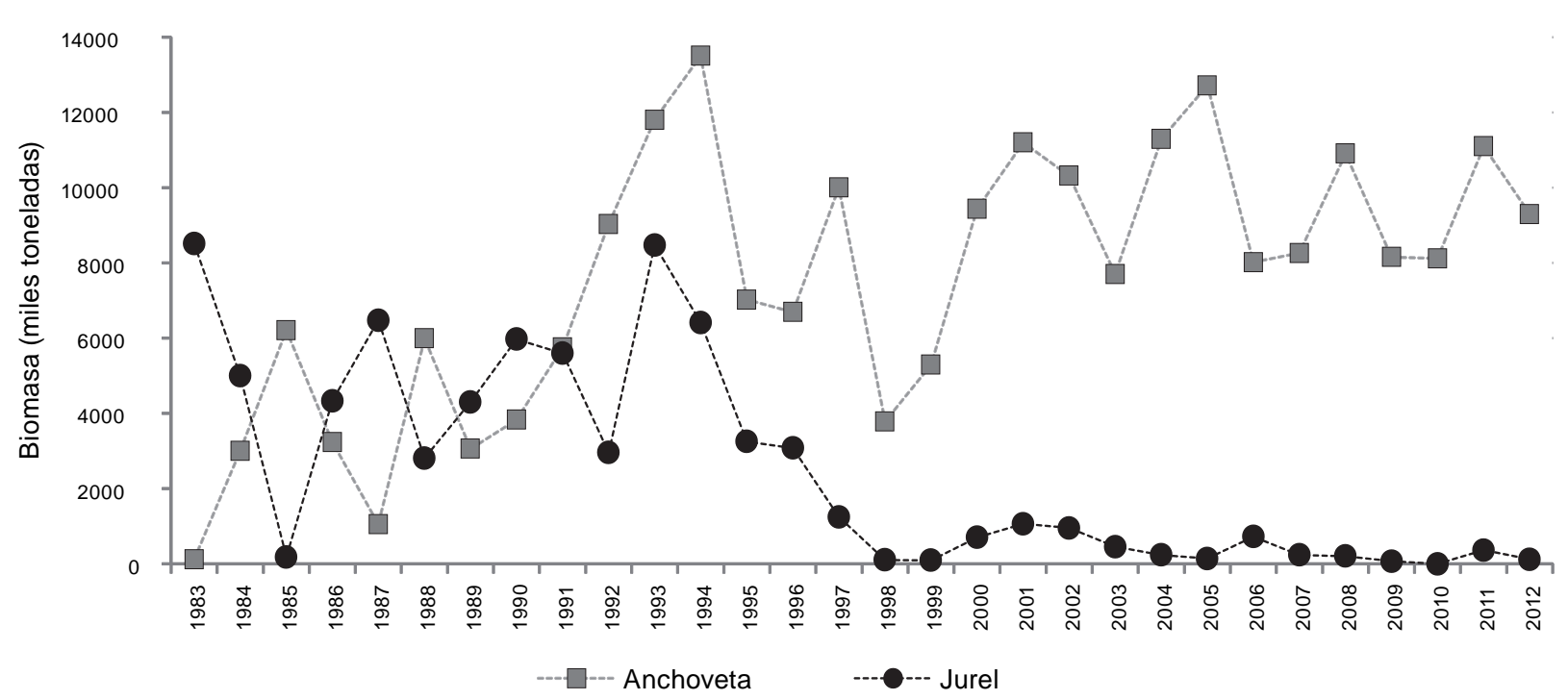

Figura 11. Estimados de biomasa acústica de jurel T. murphyi y anchoveta E. ringens en el mar peruano durante el periodo $1983-2012$.

Figure 11. Acoustic biomass estimates of Jack mackerel T. murphyi and anchovy E. ringens in Peruvian waters during the period $1983-2012$

et al. 2011, Flores et al. 2013), y también probablemente en una parte importante del Pacífico Sudoriental (Espino 2013). Estos cambios en la profundidad de la capa de mínimo oxígeno asociadas a la temperatura superficial del mar y a la salinidad superficial podrían explicar los cambios en la disponibilidad de T. murphyi y S. japonicus observados frente a la costa peruana, especialmente desde fines de los años noventa.

Variaciones en la disponibilidad espacial de jurel y otros recursos.- Se observa que las variaciones en la distribución de T. murphyi hacia las regiones centro y norte de Perú se presentan asociadas a la mayor velocidad y dirección sur-norte de las corrientes, que se producen durante e inmediatamente después de un evento La Niña. En estas circunstancias, que correspondería al patrón 2 descrito por Dioses (2013), la disponibilidad de T. murphyi es mayor y consecuentemente se incrementan los niveles de desembarque. En este sentido se destacan los altos desembarques alcanzados en el año 2001, y que también se registraron en Ecuador (Fig. 13). Algo similar se observó en los desembarques alcanzados durante eventos La Niña (Trasmonte \& Silva 2008) como en 1995-96 (Niña moderada, con una duración de 9 meses), 2001 (Niña débil, con una duración de 7 meses) y 2006-2007 (Niña fuerte, con una duración 9 meses). Un evento frío similar ocurrió en el 2010 (ENFEN, 2010) que fue seguido por una recuperación de los desembarques en el mar peruano durante el 2011 y que también tuvo repercusiones positivas en Ecuador.

\section{Sumario}

La documentación existente revela la presencia de jurel $T$. murphyi en los desembarques artesanales en Perú desde 1907. Desde los años sesenta, las estadísticas ubicaron al jurel entre las tres primeras especies de peces pelágicos en la pesquería, y partir del año 2000 se ubicó en segundo lugar después de la anchoveta $E$. ringens.

Las capturas de T. murphyi son realizadas por la flota artesanal, industrial cerquera y de altura (barcos arrastreros). Históricamente, la flota dedicada a la pesquería de anchoveta y sardina paso a capturar alternativamente T. murphyi durante sus periodos de mayor disponibilidad y en épocas de veda de E. ringens.

En el 2011, la estructura de la flota con permiso de pesca para T. murphyi estuvo constituida por 62 embarcaciones industriales de acero y 42 industriales de madera. La capacidad de bodega acumulada de toda la flota es de $33259 \mathrm{~m}^{3}$, de las cuales 30177 $\mathrm{m}^{3}$ corresponden a la flota industrial de acero y 3082 a la flota

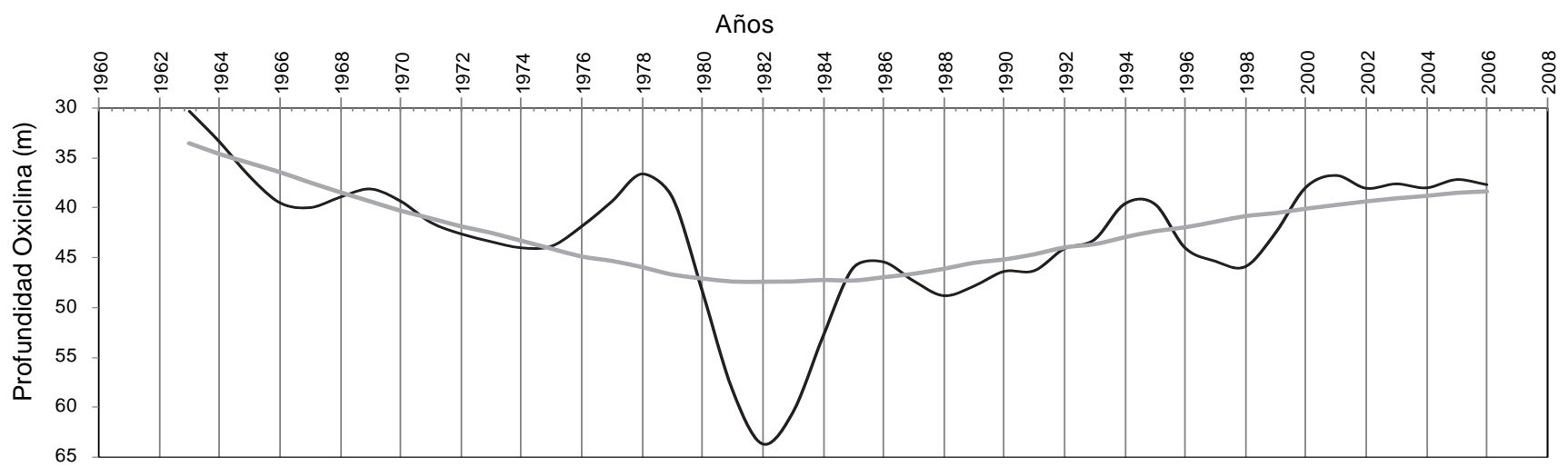

Figura 12. Profundidad de la capa de mínimo oxígeno en Perú durante 1960 - 2005, (Bertrand et al. 2011, Flores et al. 2013).

Figure 12. Depth of the layer of minimum oxygen in Peru during 1960 - 2005, (Bertrand et al. 2011, Flores et al. 2013). 


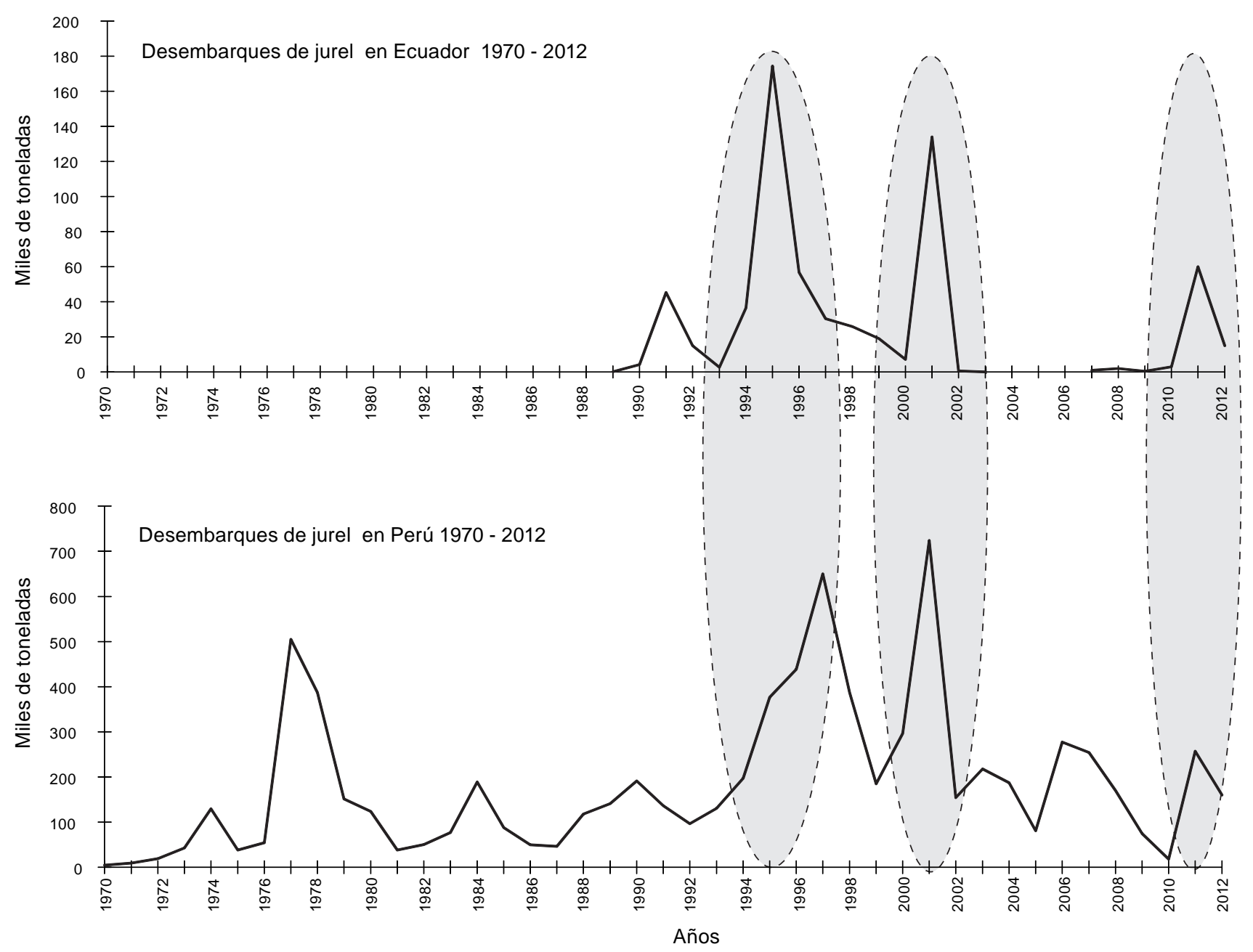

Figura 13. Desembarques de jurel T. murphyi en Perú y en Ecuador. Las áreas sombreadas indican periodos coincidentes en la mayor disponibilidad de jurel T. murphyi en Perú y Ecuador.

Figure 13. Landings of Jack mackerel T. murphyi in Peru and Ecuador. The shaded areas indicate matching periods of increased availability of Jack mackerel T. murphyi in Peru and Ecuador.

industrial de madera.

Las capturas de T. murphyi tuvieron un notable incremento a partir de 1972, alcanzando los máximos valores en 1977, 199697 y 2001. Posteriormente se observó una tendencia descendente con el mínimo valor en 2010. Sin embargo, en 2011 aumentó la disponibilidad de jurel, alcanzádose una captura cercana a las 260000 toneladas, superior a lo obtenido en los años 2007 y 2008 y que podría indicar un cambio en la tendencia descendente observada.

En el periodo 1970 - 2002 las capturas de T. murphyi presentaron los mayores valores en verano y primavera, mientras que en los últimos años (2003 - 2011) la mayor disponibilidad estacional se ha limitado al verano.

Las capturas mensuales de T. murphyi efectuadas por la flota industrial y artesanal en el periodo 1997 - 2011 mostraron tendencias similares, con periodos de mayor disponibilidad en 2001 y entre los años 2006 a 2008. Las capturas de T. murphyi en la parte norte (Máncora-Pimentel y Máncora-Chimbote) fueron mayores antes del 2002, mientras que en la parte centrosur (San Juan-Ilo y Huacho-Ilo) las capturas fueron mayores después del 2001.

En las capturas efectuadas por la flota cerquera se registran la presencia conjunta de los recursos jurel T. murphyi y caballa $S$. japonicus, en porcentaje promedio de $72 \%$ y $28 \%$ respectivamente.

La persistencia de anomalías negativas de la temperatura superficial del mar observada en los años 1977, 1996-97 y 2001 favoreció la mayor disponibilidad de T. murphyi y menor de $E$. ringens en las regiones centro y sur.

La distribución de T. murphyi en las regiones centro y norte de Perú están influenciadas por la mayor velocidad y dirección sur-norte de las corrientes, que se producen durante e inmediatamente después de un evento La Nińa, incrementando la disponibilidad de T. murphyi en las regiones centro y norte del Perú llegando a registrarse inclusive, desembarques en el Ecuador.

\section{Literatura citada}

Bertrand A., M. Segura, M. Gutiérrez \& L. Vásquez. 2004. From small-scale habitat loopholes to decadal cycles: a habitat-based hypothesis explaining fluctuation in pelagic fish populations off Peru. Fish and Fisheries 5: 296 - 316. DOI: 10.1111/j.1467-2679.2004.00165.x

Bertrand A., A. Chaigneau, S. Peraltilla, J. Ledesma, M. Graco. 2011. Oxygen: A Fundamental Property Regulating Pelagic Ecosystem Structure in the Coastal Southeastern Tropical Pacific. PLoS ONE 6(12): e29558. DOI:10.1371/journal.pone.0029558.

Bouchon M., S. Cahuin, E. Diaz, M. Niquen. 2000. Captura y esfuerzo pesquero de la pesquería de anchoveta peruana (Engraulis ringens). Bol. Int. Mar Perú Vol. 19 (1 y 2): 109- 116. 
Coker R.E. 1907. Condición en que se encuentra la pesca marina desde Paita hasta bahía de la Independencia. Boletín del Ministerio de Fomento, Año VI, No 2, 30 de noviembre de 1907, 89-117.

Coker R.E. 1910. Condiciones de la pesquería de Mollendo. Boletín del Ministerio de Fomento, Año VIII, No 8, 31 de octubre de 1910, 64-114.

DS $N^{\circ}$. 001-2002-PRODUCE. 2002. Establecen que los recursos sardina, jurel y caballa serán destinados al consumo humano directo. 06 de setiembre 2002. El Peruano. Normas Legales: 229522-229523.

DS N ${ }^{\circ}$. 011-2007-PRODUCE. 2007. Aprueban Reglamento de Ordenamiento Pesquero de Jurel y Caballa. 13de abril de 2007. El Peruano Normal Legales: 343399-343402.

Dioses T. 2013. Patrones de distribución y abundancia del jurel Trachurus murphyi en el Perú. En: Csirke J., R. Guevara-Carrasco \& M. Espino (Eds.). Ecología, pesquería y conservación del jurel (Trachurus murphyi) en el Perú. Rev. peru. biol. número especial 20(1): 067- 074

Flores R., M. Espino, G. Luque \& J. Quispe. 2013. Patrones de variabilidad ambiental en el mar peruano. En: Csirke J., R. Guevara-Carrasco \& M. Espino (Eds.). Ecología, pesquería y conservación del jurel (Trachurus murphyi) en el Perú. Rev. peru. biol. número especial 20(1): $021-028$

Espino M. 2013. El jurel Trachurus murphyi y las variables ambientales de macroescala. En: Csirke J., R. Guevara-Carrasco \& M. Espino (Eds.). Ecología, pesquería y conservación del jurel (Trachurus murphyi) en el Perú. Rev. peru. biol. número especial 20(1): 009-020

ENFEN. 2010. Nota de Prensa No 08 del Comité Multisectorial encargado del Estudio Nacional del Fenómeno El Niño (ENFEN). 03 de agosto de 2010 .
Gutiérrez M., P.R. Castillo, F. Ganoza, N. Herrera, S. Peraltilla \& A. Aliaga. 2004. Análisis de la distribución y abundancia de algunos recursos pelágicos peruanos acústicamente evaluados en la primavera 2001. Inf. Inst. Mar Perú, 32(2): 155-165.

Grechina A. 1998. Historia de investigación y aspectos básicos de la ecología del jurel Trachurus symmentricus murphyi (Nichols) en alta mar del Pacífico Sur. En: D. Arcos (Ed.). Biología y ecología del jurel en aguas chilenas, Instituto de Investigaciones Pesqueras S.A. Talcahuano, Chile: 11-34.

Jordán R. 1983. Variabilidad de los recursos pelágicos en el Pacífico Sudeste. En G.D. Sharp y J. Csirke (Eds.), Actas de la Consulta de Expertos para examinar los cambios en la abundancia y composición por especies de recursos de peces neríticos. San José, Costa Rica, 18-29 abril de 1983. Una reunión preparatoria para la Conferencia Mundial de la FAO sobre ordenación y desarrollo pesqueros. FAO Fish.Rep./ FAO, Inf. Pesca (291) Vol. 2:113-129.

Niquen M., M. Bouchon, S. Cahuín \& E. Díaz. 200o. Pesquería de anchoveta en el mar peruano. Bol. Inst. Mar Perú Vol. 19 (1 y 2): 117- 124.

Tilic I. 1963. Material estadístico sobre la industria peruana de harina de pescado. Inf. Inst. Inv. Rec. Marinos $\mathrm{N}^{\circ} 14,51 \mathrm{p}$.

Trasmonte G., \& Y. Silva. 2008. Evento La Niña: Propuesta de definición y clasificación según las Anomalías de Temperatura Superficial del Mar en el Área Niño 1+2. Inf. Inst. Mar Perú 35(3): 199-207.

Tsukayama I. 1983. Recursos pelágicos y sus pesquerías en Perú. Rev. Com. Perm. Pacífico Sur 13: 25-63.

Zuta S. 1983. El ambiente marino y las fluctuaciones de las principales poblaciones pelágicas de la costa peruana. En: G.D. Sharp y J. Csirke (Eds.), Actas de la Consulta de Expertos para examinar los cambios en la abundancia y composición por especies de recursos de peces neríticos. San José, Costa Rica, 18-29 abril 1983. Una reunión preparatoria para la Conferencia Mundial de la FAO sobre ordenación y desarrollo pesqueros. San José, Costa Rica, 18 - 29 April 1983. FAO Fish. Rep. 291(2): 179-254. 